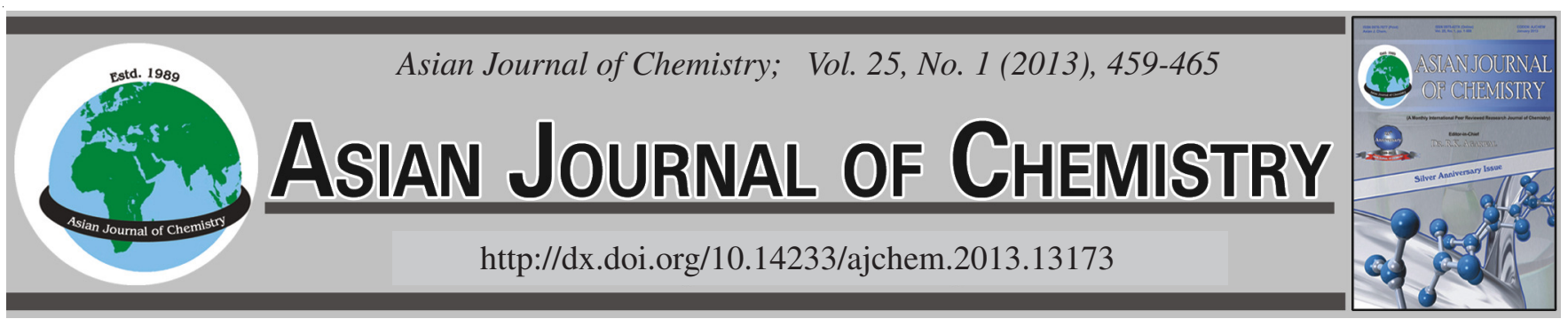

\title{
Molecular Encapsulation of Lavender Essential Oil by $\beta$-Cyclodextrin and Dimethyl Dihydroxy Ethylene Urea for Fragrance Finishing of Cotton Fabrics
}

\author{
R. KhAJAVI ${ }^{1, *}$, M. Ahrari ${ }^{2}$, T. TOLIYAT ${ }^{3}$ and L. BAHADORI ${ }^{1}$
}

\begin{abstract}
${ }^{1}$ Department of Textile Engineering, South Tehran Branch, Islamic Azad University, Tehran, Iran
${ }^{2}$ Department of Textile Engineering, Science and Research Branch, Islamic Azad University, Tehran, Iran

${ }^{3}$ Pharmaceutical Department, Tehran University of Medical Sciences, Tehran, Iran

*Corresponding author: Tel: +98 912 2107490; E-mail: khajavi@azad.ac.ir
\end{abstract}

(Received: 3 December 2011;

Accepted: 30 July 2012)

AJC-11900

Volatility of fragrance compounds on aroma textiles is considered as a deficiency by producers and consumers. Molecular encapsulation
can be used for preserving the fragrance compound as the guest molecule so prolonging the fragrance of the textiles. In this study
molecular encapsulation by $\beta$-cyclodextrin $(\beta-\mathrm{CD}$ ) applied for hosting of lavender essential oil (LEO) on cotton fabric. Dimethyl dihydroxy
ethylene urea also used for bounding the $\beta$-cyclodextrin to cotton fabric. Phenolphthalein and spectrophotometric methods used for the
detection and measurement of $\beta$-cyclodextrin, lavender and the durability of fragrance finishing on cotton fabric samples. Lavender
essential oil bonded to $\beta$-cyclodextrin according to their mutual hydrophobic character and treated samples with molecular encapsulated
fragrance compound showed more durability in comparison with untreated and dimethyl dihydroxy ethylene urea treated samples.

Key Words: Molecular encapsulation, $\beta$-Cyclodextrin, Fragrance textiles, Lavender essential oil, Phenolphthalein method, Dimethyl dihydroxy ethylene urea.

\section{INTRODUCTION}

Molecular encapsulation in supramolecular chemistry is the confinement of a guest molecule inside the cavity of a supramolecular host molecule (molecular capsule, molecular container or cage compounds). Molecular container compounds provide a new space for reaction chemistry, both literally and figuratively, through the encapsulation of smaller guest molecules. Fiedler, Bergman and Raymond reported the stabilization of reactive organometallic intermediates in aqueous solution through molecular encapsulation ${ }^{1}$.

In supramolecular chemistry, host-guest chemistry describes complexes that are composed of two or more molecules or ions that are held together in unique structural relationships by forces other than those of full covalent bonds. The stabilization of reactive species within the narrow confines of a host cavity is an intriguing functional property of covalent and self-assembled hosts and has been observed in a number of different systems. Both kinetic and thermodynamic factors can contribute to this stabilization: the tight host portals may kinetically inhibit guest escape and subsequent reactivity or strong host-guest binding may stabilize an otherwise reactive structure $^{1}$.

Common host molecules are cyclodextrins, calixarenes, cucurbiturils, porphyrins, metallacrowns, crown ethers, zeolites, cyclotriveratrylenes, cryptophanes and carcerands. Cyclodextrins are able to form host-guest complexes with hydrophobic molecules given the unique nature imparted by their structure. As a result, these molecules have found a number of applications in a wide range of fields. It is shown that, for any host-guest system, a rough screening of the most probable complex stoichiometries can be obtained in a model free form, using only calorimetric data ${ }^{2}$. The outer surface of these doughnut-shaped molecules is hydrophilic, but they possess an axial open cavity, which is of hydrophobic character and capable of including other apolar molecules (or their moiety) in case of geometric compatibility. This is the essence of molecular encapsulation by inclusion complex formation ${ }^{3}$. Cyclodextrins are a family of cyclic oligosaccharides composed of $\alpha-(1,4)$ linked glucopyranose subunits ${ }^{4}$.

Lavender can be mentioned as a guest molecule. Lavender oil is an essential oil obtained by distillation from the flower spikes of certain species of lavender. It is a complex mixture of naturally occurring phytochemicals, including linalool and linalyl acetate which their chemical structure brought in Fig. 1. Lavender essential oil is popular as a complementary medicine in its own right and as an additive to many over the counter complementary medicine and cosmetic products. The oil is traditionally believed to have sedative, carminative, antidepressive and anti-inflammatory properties, in addition to its 


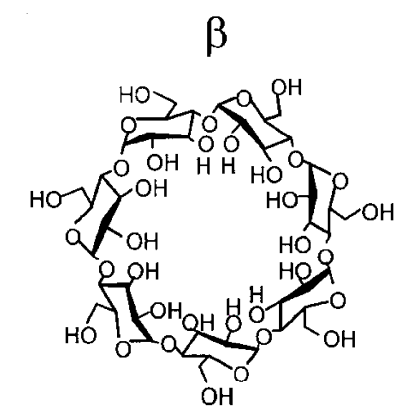

(a)

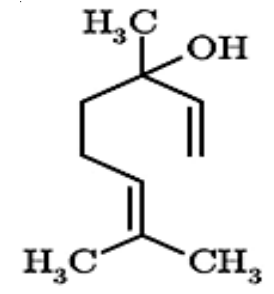

(c)

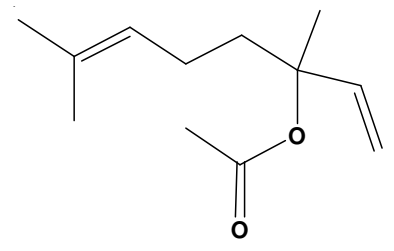

(b)

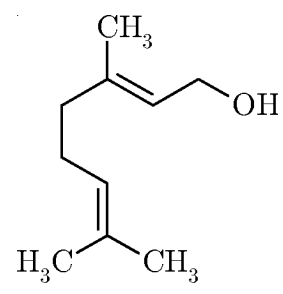

(d)
Fig. 1. Chemical structures of a) $\beta$-Cyclodextrin, b) Linalyl acetate, c) Linalool and d) Geraniol ${ }^{45}$

recognized antimicrobial effects ${ }^{5}$. Hashimoto ${ }^{6}$ reviewed the application of cyclodextrins in the food, cosmetic and textile industrial fields as solubilizing agents, stabilizers and emulsifiers.

Cyclodextrins can serve as multi-functional drug carriers, through the formation of inclusion complex or the form of cyclodextrin/drug conjugate and, thereby potentially serving as novel drug carriers ${ }^{7}$. Sangalli et al. ${ }^{8}$ investigated the release behaviour of poorly soluble drugs (naproxen and ketoprofen) from inert (acrylic resins) and hydrophilic swellable (highviscosity hydroxypropylmethylcellulose) tableted matrices containing $\beta$-cyclodextrin. Cyclodextrins enhance topical drug delivery by increasing the drug availability at the barrier surface and it appears that cyclodextrins can only enhance topical drug delivery in the presence of water'.

Lee et al. ${ }^{10}$ investigated the possibility of textile finishing of cyclodextrin containing cotton fibers using benzoic acid as an antibacterial finishing agent or vanillin as an aroma finishing agent. Grechin, Buschmann, Schollmeyer investigated the coordination and adsorption properties of cellulose materials with fixed monochlorotriazine substituted $\beta$-cyclodextrin for different primary, secondary and tertiary volatile aliphatic amines $^{11}$. Cireli and Yurdakul ${ }^{12}$ studied the applicability of cylcodextrins in textile dyeing and washing processes. They used $\beta$-cyclodextrin in direct dyeing of cellulosic fabrics and in rinsing processes of direct dyed fabrics. Szejtli stated that in the future cyclodextrins might play a significant role in the textile industry and might be used: (1) to remove surfactants from washed textiles; (2) to substitute surfactants; (3) in finishing textiles; (4) when bound chemically to fibers, to provide enhanced hydrophilicity and first of all, inclusion complex forming ability to immobilize perfumes, insect repellents and antimicrobial agents ${ }^{13}$. Cyclodextrins have been used for the separation of isomeric hydrocarbons, enrichment of unsaturated fatty acids and the removal of organic solvent vapours from air, toxic substances from industrial wastes, bitter components from citrus juices and caffeine from coffee, among other applications ${ }^{14}$. Voncina and Le Marechal crosslinked $\beta$-cyclodextrin molecules on hydroxyl groups of cellulose via BTCA $^{15}$. Four- to ten-fold improvement of colour uniformity and minor changes of colour yield have been found upon dyeing Nylon 66 and microfiber Nylon 6 fabrics in the presence of cyclodextrin compared to dyeing without it ${ }^{16}$. Krzysztof and Katarzyna summarized the results of recent studies on the application of parent cyclodextrins $\left(\alpha-, \beta-, \gamma_{-}\right)$and some of their derivatives in topical formulations. General properties, current legal status and toxicological aspects of cyclodextrins were briefly described. The goal of using cyclodextrins to create new formulations with well-known actives, advantages and limitations in topical formulations was presented and possible applications in such preparations were also discussed ${ }^{17}$. Cyclodextrins can increase oral bioavailability, stabilize compounds to chemical and enzymatic degradation and can affect permeability through biological membranes under certain circumstances ${ }^{18}$. Loftsson and Brewster discussed the effects of substitution on various cyclodextrin properties and the forces involved in the drug-cyclodextrin complex formation ${ }^{19}$.

$\beta$-Cyclodextrin had been used as a drug release candidate in pharmaceutical application ${ }^{20}$. Buchbauer studied and investigated the methods in aroma therapy ${ }^{21}$. The use of surfaceactive agents in finishing can also have a negative effect if residual layers are deposited (modified dyeing behaviour, printing faults). Buschmann et al. ${ }^{22}$ studied whether these deposits can be effectively removed by complexing surfaceactive agents with cyclodextrins. They also stated that new areas of applications of cyclodextrins with textiles are also possible ${ }^{23}$. Breteler et al. ${ }^{24}$ focused on the opportunities of textiles bearing cyclodextrins, aza-crown ethers or fullerenes, as well as ion-exchange fibres, drug-loaded hollow fibres, textiles treated with nanoparticles and fibres with bioactive compounds in their embodiment, also the delivery systems had been discussed and compared in terms of biostability, biodegradability, controllability, toxicity, carcinogenicity, interface reactions, material costs and the fabrication process. Nostro et al. ${ }^{25}$ were permanently grafted $\beta$-cyclodextrin onto the surface of a cellulosic fabric, namely Tencel® according to different procedures. A new microencapsulation technology with roots in the textile industry, yeast based microencapsulation, were described ${ }^{26}$. A research attempt at obtaining aroma therapic textiles carried out, together with the broad background of research into aromachology investigation ${ }^{27}$. The suitable technical conditions of forming stable $\beta$-cyclodextrin sol-gel solution were presented by Wang and $\mathrm{Chen}^{28}$. The effects of the nature of essential oils, such as detection threshold and tenacity on the fragrance-release property were discussed ${ }^{29}$. The possibility of using cyclodextrins in Crease Resistant Finish evaluated by Kut et al. ${ }^{30}$. The phenolphthalein solution used in the assay of $\beta$-cyclodextrin is not stable and undergoes spontaneous decolourization. A simple procedure was described by Goel et al. to circumvent this problem allowing reproducible and reliable results to be obtained ${ }^{31}$. The production of woven and knitted fabrics with aroma therapy characteristics targeted by applying on their surface chitosan-lavender essential oil and monochlorotriazinyl- $\beta$-cyclodextrin-lavender essential oil systems $^{32}$. Multiple patents describe repellent compositions for use in topical agents, cosmetic products, incense, fumigants, indoor and outdoor sprays, fibers, textiles among other applications ${ }^{33}$. 
The cleaner technology (CT) concept was applied to the household silk dyeing process, to develop a more environmentally friendly silk product by Poonprasit et al. ${ }^{34}$ Boh and Knez reported the development and testing of three types of microcapsules for applications in textile products, namely microencapsulation of antimicrobial essential oils of sage, lavender and rosemary for nonwoven textile shoe insoles ${ }^{35}$. The possibility to achieve the insecticide properties of the textile substrates treated with ecological friendly insecticide reagents had been studied by Vraz et al. ${ }^{36}$. The textile fabric surface functionalized with MCT- $\beta$-cyclodextrin through covalent bonding. After the treatment of modification, different UV protective chemical substances were "loaded" by spraying their solutions on the cyclodextrin-functionalized fibers and the results had been further investigated ${ }^{37}$. Molecular encapsulation of various apolar compounds with $\alpha$-cyclodextrin $(\alpha-$ $\mathrm{CD}$ ) is becoming a widely applied technique to produce food, pharmaceutical and agricultural materials. Encapsulated ethylene in the form of inclusion complexes (ICs) with cyclodextrin, which is in powder form, could be used in fruit ripening and other aspect of plant growth regulation. Ethylene was complexed with an $\alpha$-cyclodextrin under 0.2-1.5 MPa for 12-120 h by Ho et al. ${ }^{38}$. Buschmann et al. ${ }^{39}$ reviewed cyclodextrins and their applications in textiles. Methods for enhancing bioavailability of drug had been reviewed by Moon et al. $^{40}$. Understanding the interactions of cyclodextrins (CDs) and cucurbit[n] uril (CB[n]) hosts with a variety of guest molecules following their encapsulation within the cavity of these macrocycles have become increasingly important in the recent years. The molecular electrostatic potential (MESP) topography has been utilized to obtain the 'effective' cavity diameter and height of $\mathrm{CB}[\mathrm{n}](\mathrm{n}=6-8)$ homologues and 8 conformers each of $\alpha$-, $\beta$ - and $\gamma$-cyclodextrin. It has been shown that the shape of cyclodextrin cavity be in cone- or barrel-like stems from the hydrogen bonding patterns within primary hydroxyl groups ${ }^{41}$. Lavender oil can be effectively used in finishing of fabrics including pure cotton fabric as it offers a number of advantages in medical applications such as anxiety, stress, insomnia and alopecia. Lavender oil aroma therapy reduces the level of perceived anxiety in nursing students and offers an increased sense of well being. The lavender oil based finish also offers 15 wash durability ${ }^{42}$. $\beta$-Cyclodextrine/benzoic acid complex was prepared and reacted with cyanuric chloride (2,4,6-trichloro-1,3,5-triazine). Identification of complex formation and reaction was checked by FT-IR UV-VIS and EDX. By reacting this material with cotton fiber, the deodourant fiber was prepared by Choi et $a l .{ }^{43}$. Cernat et al. studied of application certain chemical principles of modification the textile material characteristics which are based on the permanent bonding of some supramolecular combinations.

Textile industry needs an evolution in the near future due to consumer expectations and producers competitions. Cyclodextrins have shown interesting abilities in this field, but the blockbuster will be the chemical binding of cyclodextrins onto surface of the natural and synthetic fibres. The immobilized cyclodextrins can bind volatile molecules like the unpleasant components of sweat, or cigarettes smoke, or when previously charged, the slow release of fragrances, insect repellents or even drugs (for transdermal delivery) will be possible by wearing bonded cyclodextrin containing garments.

According to afore mentioned literature survey, in this study molecular encapsulation by $\beta$-cyclodextrin applied for hosting of lavender essential oil on cotton fabric. Dimethyl dihydroxy ethylene urea (Fig. 2) used for bounding the $\beta$ cyclodextrin to cotton fabric. Phenolphthalein and spectrophotometric methods used for the detection and measurements of $\beta$-cyclodextrin, lavender and the durability of fragrance finishing on cotton fabric samples.

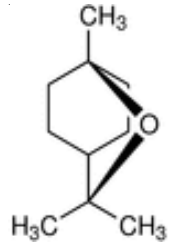

(a)

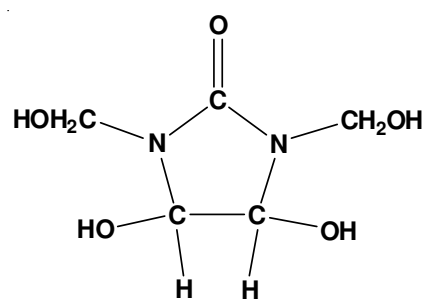

(b)
Fig. 2. Chemical structures of (a) 1,8-Cineole, (b) Dimethyl dihydroxy ethylene urea

\section{EXPERIMENTAL}

Woven twill cotton fabric $\left(140 \mathrm{~g} / \mathrm{m}^{2}\right)$ was supplied by Ardakan Company Yazd, Iran. $\beta$-Cyclodextrin was provided by Merck, Germany (m.w. = 1135 g/mol). LEO (Guangzhou $\mathrm{H} \& \mathrm{C}$ fragrances Co. Ltd: EMPEROR) supplied by H\&C fragrances Co. Ltd., Guangzhou, China. 1,3-Dimethylol-4,5dihydroxyethylene urea (DMDHEU; Fixapertcpconc;) supplied by BASF. All other chemicals were analytical grades and provided by Merck and Fluka.

Preparing cotton fabric samples: Cotton fabric desized (soda boiled for $0.5 \mathrm{~h}$ ) and after assurance of no residual of starch on fabric by starch test method $(5 \mathrm{~g}$ potassium iodide, $2 \mathrm{~g}$ iodine dissolved in $1 \mathrm{~L}$ ). The fabric was scoured, dried and cut into $10 \times 10 \mathrm{~cm}^{2}$ samples.

Fragrance finishing of samples: In order to compare the effects of $\beta$-cyclodextrin and DMDHEU on the reservation of lavender essential oil on cotton fabric, four series of samples prepared. They had been sprayed with three different solutions in order to get to $70 \%$ weight increase according to Table-1, then dried and cured. For second series of samples, lavender first combined with $\beta$-cyclodextrin in a separate bath. An emulsion of water and alcohol with 1:3 proportions prepared, stirred for $5 \mathrm{~min}$ at $4000 \mathrm{rpm}$ and the contents were transferred to an enclosed container. The essential oil was added to emulsion over $0.5 \mathrm{~h}$ and stirred for $2 \mathrm{~h}$ at $40{ }^{\circ} \mathrm{C}$.

Spectroscopic analysis: Alcoholic solution of lavender (con. $0.26 \mathrm{~g} / \mathrm{L}$ ) prepared, then its absorption values under a spectrum of wavelength determined and hereby the $\lambda_{\max }$ obtained.

For concentration determination of the lavender, absorptions of lavender with different concentrations $(0.1,0.2,0.3$ and $0.4 \mathrm{~g} / \mathrm{L}$ ) with fixed $\lambda_{\max }$ were obtained by the spectrophotometer. The linear concentration-absorbance calibration curve and linear (first-order) equation obtained. 
TABLE-1

TREATMENTS CONDITIONS OF FOUR SERIES OF SAMPLES

\begin{tabular}{|c|c|c|c|c|}
\hline $\begin{array}{l}\text { Sample } \\
\text { No. }\end{array}$ & Spraying solution (Pick up $70 \%$ ) & $\begin{array}{c}\text { Drying for } \\
5 \text { min at } 80^{\circ} \mathrm{C}\end{array}$ & $\begin{array}{c}\text { Curing for } \\
5 \mathrm{~min} \text { at } 150{ }^{\circ} \mathrm{C}\end{array}$ & Immersion solution $\left(5 \mathrm{~min}\right.$ at $\left.80^{\circ} \mathrm{C}\right)$ \\
\hline 1 & $40 \mathrm{~g} / \mathrm{L} \mathrm{DMDHEU}, 8 \mathrm{~g} / \mathrm{L} \mathrm{MgCl}_{2}, 2 \mathrm{~g} / \mathrm{L} \beta-\mathrm{CD}$ & + & + & $0.28 \% \mathrm{~g} / \mathrm{L}$ Lavender in alcohol \\
\hline 2 & $40 \mathrm{~g} / \mathrm{L} \mathrm{DMDHEU}, 8 \mathrm{~g} / \mathrm{L} \mathrm{MgCl}_{2}$ & + & + & $0.28 \% \mathrm{~g} / \mathrm{L}$ Lavender combined by $\beta-\mathrm{CD}$ \\
\hline 3 & $40 \mathrm{~g} / \mathrm{L} \mathrm{DMDHEU,} 8 \mathrm{~g} / \mathrm{L} \mathrm{MgCl}_{2}$ & + & + & $0.28 \% \mathrm{~g} / \mathrm{L}$ Lavender in alcohol \\
\hline 4 & $0.28 \mathrm{~g} / \mathrm{L}$ Lavender in ethanol media & + & - & - \\
\hline
\end{tabular}

Phenolphthalein test: Solution of $4 \mathrm{mM}$ phenolphthalein in alcohol and $125 \mathrm{mM}$ sodium carbonate in distilled water prepared $(\mathrm{pH}=7)$. $1 \mathrm{~mL}$ of phenolphthalein, $4 \mathrm{~mL}$ ethanol solution added to $100 \mathrm{~mL}$ sodium carbonate solution ${ }^{31}$. Different amounts of $\beta$-cyclodextrin added to previously prepared solution and the calibration curve obtained. Samples placed in 4 baths for $0.5 \mathrm{~h}$, removed and absorbance of the existed waste was measured. Amount of the existed $\beta$-cyclodextrin was calculated.

Durability of fragrance fabric samples: Fragrance fabrics were kept at ambient temperature for 14 days. For quantitative evaluation samples added to alcohol bath and retained for $0.5 \mathrm{~h}$. The concentration of lavender was measured by the spectrophotometer and phenolphthalein test was carried out in order to identify the existence of $\beta$-cyclodextrin.

Lavender absorption calibration curve: Four standard concentrations of lavander essential oil prepared with wateralcohol solution and their absorbance obtained at $211 \mathrm{~nm}$ wavelength $\left(\lambda_{\max }\right)$ (Table-2 and eqn. 1):

$$
\mathrm{Y}=7.7041 \mathrm{x}+0.000
$$

$\mathrm{Y}=$ absorbance, $\mathrm{x}=$ concentration of lavender.

\begin{tabular}{ccc}
\multicolumn{3}{c}{ TABLE-2 } \\
OBTAINED ABSORBANCE AMOUNTS \\
FROM SPECTROPHOTOMETER \\
\hline Standard & Concentration $(\mathrm{g} / \mathrm{L})$ & Absorbance \\
\hline 1 & 0.1 & 0.7703 \\
2 & 0.2 & 1.5407 \\
3 & 0.3 & 2.3110 \\
4 & 0.4 & 3.0814 \\
\hline
\end{tabular}

\section{RESULTS AND DISCUSSION}

Length and volume of lavander essential oil components were measured to investigate the feasibility of placing lavender in $\beta$-cyclodextrin. Chemical structure of linalool molecule which is one of the main constituents of lavander essential oil with specified bonds angles and lengths are shown in Fig. 3 and Table-3.

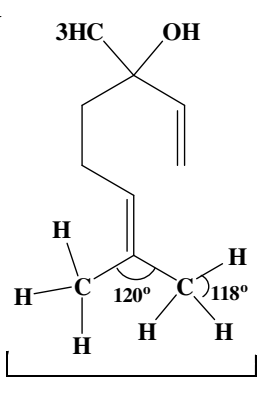

$$
\text { L }
$$

Fig. 3. Structure of linalool and its bond length and angles in hydrophobic head of molecule, L: maximum width length

\begin{tabular}{cc} 
TABLE-3 \\
DIFFERENT BONDS AND THEIR \\
BOND LENGTH IN LINALOOL MOLECULE \\
\hline Bond & Bond length $(\mathrm{pm})$ \\
\hline C-H & 109 \\
C=C & 134 \\
H-O & 96 \\
C-C & 154 \\
\hline
\end{tabular}

There is a tendency between the hydrophobic head or the hydrophobic groups of methylene $\left(-\mathrm{CH}_{3}\right)$ groups of linalool and the hydrophobic part of $\beta$-cyclodextrin molecule or its cavity. The length " $\mathrm{L}$ " which is representing the size of linalool hydrophobic tail molecule is not greater than $642 \mathrm{pm}$. It is smaller than $\beta$-cyclodextrin cavity diameter which is $650 \mathrm{pm}$, so hosting the linalool by $\beta$-cyclodextrin is possible theoretically.

The ability of $\beta$-cyclodextrin to form an inclusion complex with a guest molecule is a function of two key factors. The first is steric and depends on the relative size of the cyclodextrin to the size of the guest molecule or certain key functional groups within the guest. If the guest is the wrong size, it will not fit properly into the cyclodextrin cavity. The second critical factor is the thermodynamic interactions between the different components of the system ( $\beta$-cyclodextrin, guest, solvent). For a complex to form, there must be a favourable net energetic driving force that pulls the guest into the $\beta$-cyclodextrin.

In general, there are four energetically favourable interactions that help shift the equilibrium to form the inclusion complex: (a) The displacement of polar water molecules from the apolar cyclodextrin cavity. (b) The increased number of hydrogen bonds formed as the displaced water returns to the larger pool. (c) A reduction of the repulsive interactions between the hydrophobic guest and the aqueous environment. (d) An increase in the hydrophobic interactions as the guest inserts itself into the apolar $\beta$-cyclodextrin cavity.

Lavender adsorption spectrophotometer diagram: Three peaks are represented in the absorption curve of the lavander essential oil (Fig. 4). Maximum absorbance observed at $211 \mathrm{~nm}$ wavelength, so it is considered as maximum wavelength.

Comparison of experimented samples: The amounts of residual lavander essential oils concentrations compared in Fig. 5.

The amount of lavender absorbed to the fabric in second bath is greater than the forth bath. The results indicated that lavender molecule placed in the hydrophobic cavity of $\beta$ cyclodextrin and its durability was increased.

Dimethyl dihydroxy ethylene urea bounds with hydroxyl groups of cellulose and $\beta$-cyclodextrin. Samples kept in ambient for 14 days, placed in alcohol for $0.5 \mathrm{~h}$ and the 


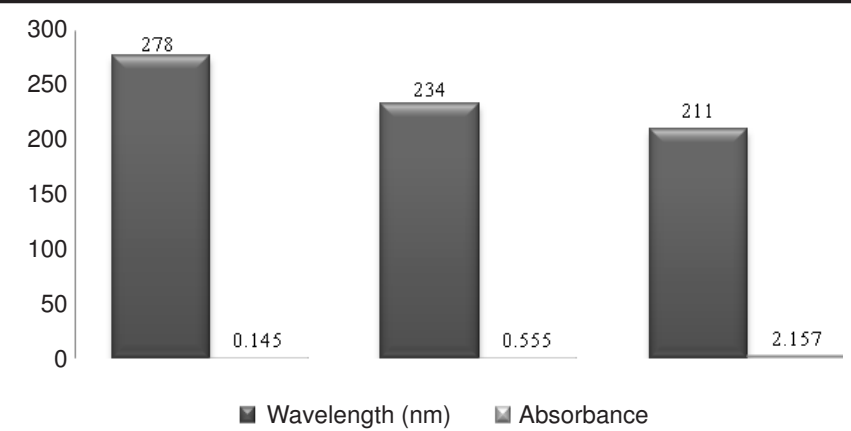

Fig. 4. Three maximum absorbance peeks of lavender

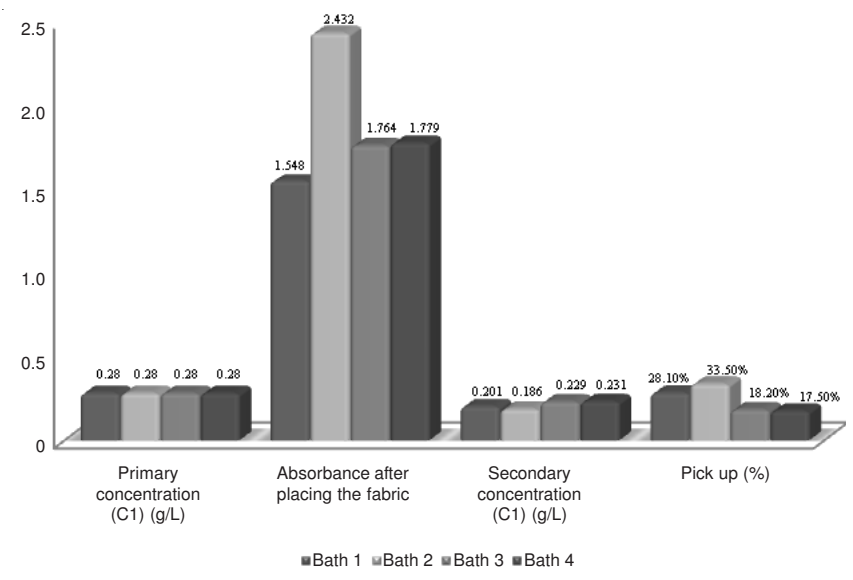

Fig. 5. Comparison of samples concentrations and pick up

absorbance amounts obtained. The amounts of absorbance inserted in eqn. 1 and the results represented in Fig. 6.

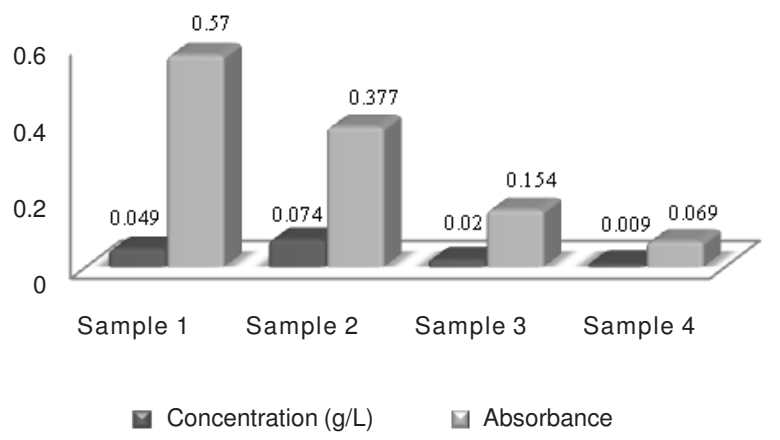

Fig. 6. Comparison of the waste of samples after placing in alcohol
The results indicated that sample 1 had the maximum lavender concentration.

$\boldsymbol{\beta}$-Cyclodextrin absorption calibration curve: The calibration curve of $\beta$-cyclodextrin absorption as a function of its concentration based on four phenolphthalein solutions with different amounts of $\beta$-cyclodextrin (1, 2, 3 and $4 \mathrm{~g} / \mathrm{L})$ obtained and amounts of absorbances are summarized in Table-4.

\begin{tabular}{|c|c|}
\hline \multicolumn{2}{|c|}{$\begin{array}{c}\text { TABLE-4 } \\
\text { OBTAINED ABSORBANCE AMOUNTS } \\
\text { FROM CALIBRATION CURVE }\end{array}$} \\
\hline Concentration $(\mathrm{g} / \mathrm{L})$ & Absorbance \\
\hline 1 & 0.2967 \\
\hline 2 & 0.3759 \\
\hline 3 & 0.5125 \\
\hline 4 & 0.6021 \\
\hline
\end{tabular}

Linear (first-order) equation obtained from Fig. 5 (eqn. 2).

$$
\mathrm{Y}=0.10531 \mathrm{x}+0.18352
$$

$\mathrm{Y}=$ absorbance, $\mathrm{x}=$ lavender concentration

Remained amount of $\boldsymbol{\beta}$-cyclodextrin on fabric: Treated samples with $\beta$-cyclodextrin were immersed in phenolphthalein solutions for $0.5 \mathrm{~h}$, then samples brought out and the absorbance of the solution determined and summarized in Table-5. Phenolphthalein acts as a guest in 'host-guest' reactions and places in the internal cavity of $\beta$-cyclodextrin, as shown in Fig. 7 a complex forms between $\beta$-cyclodextrin and phenolphthalein and causes their solution to decolourize.

\begin{tabular}{ccc}
\multicolumn{3}{c}{ TABLE-5 } \\
OBTAINED ABSORBANCE VALUES \\
\hline Sample & Concentration $(\mathrm{g} / \mathrm{L})$ & Absorbance \\
\hline 1 & 0.403 & 0.2259 \\
2 & 0.472 & 0.2332 \\
\hline
\end{tabular}

Effect of DMDHEU: DMDHEU bounds with cellulose. Four hydroxyl groups provide the bounding capability and also DMDHEU can bound with $\beta$-cyclodextrin. It has two methylol groups, one of them cross-links with cellulose and the other one bounds with $\beta$-cyclodextrin, hydroxyl groups of DMDHEU have tendency of providing hydrogen bonds with cellulose and $\beta$-cyclodextrin which are the main reason of choosing DMDHEU in this experiment (Fig. 8a-c).
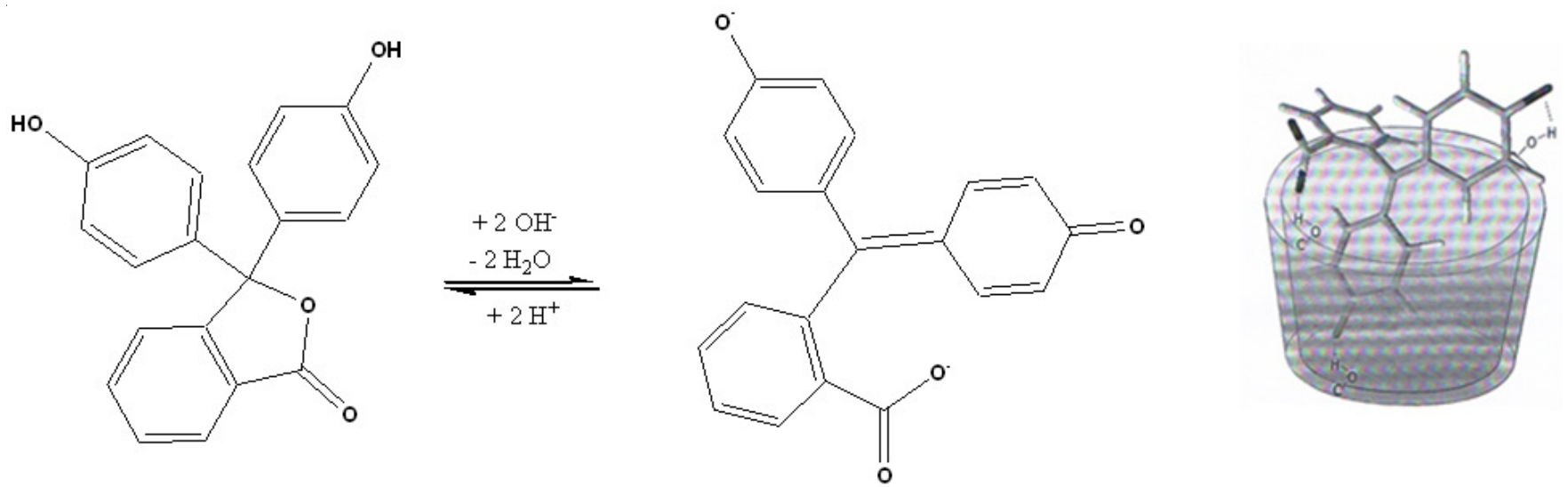

Fig. 7. (a) Phenolphthalein, (b) Phenolphthalein after dehydration, (c) Placing of phenolphthalein in $\beta$-cyclodextrin 
a)<smiles>O=C1N(CO)C(O)C(O)N1CO</smiles><smiles>[In][C+]1[C+]C=C1</smiles><smiles>O=C1N(CO)C(O)C(O)N1C[OH2+]</smiles><smiles>[CH-]=C</smiles>

b)<smiles>[CH2+]N1C(=O)N(CO)C(O)C1O</smiles>

Carbonium<smiles>O=C1N(CO)C(O)C(O)N1COC(Cl)(Cl)Cl</smiles>

c)

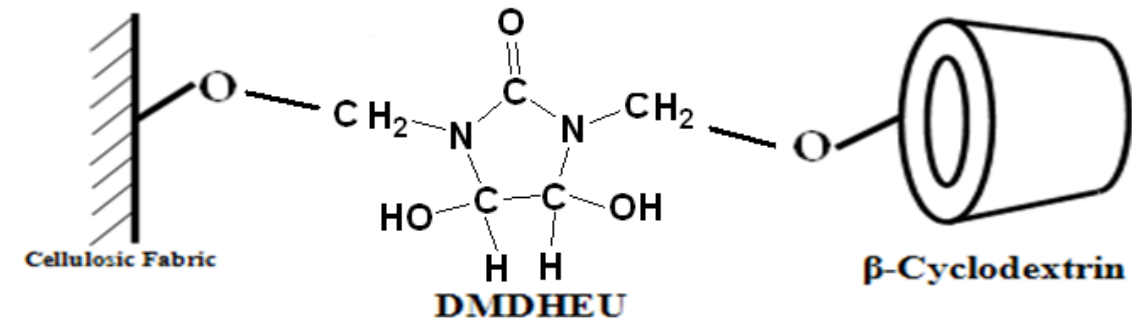

Fig. 8. (a), (b), (c) Bounds formation between DMDHEU, cellulosic fabric and $\beta$-cyclodextrin

Placing of lavender in the empty cavity of $\beta$-cyclodextrin: Volume of lavenders' constituents were greater than the volume of $\beta$-cyclodextrins' empty cavity, so lavender could not totally fit in the empty cavity of $\beta$-cyclodextrin (Fig. 9).

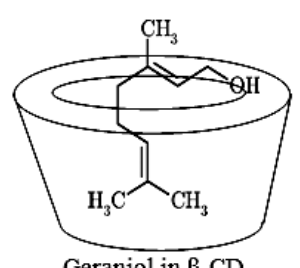

Geraniol in $\beta-C D$

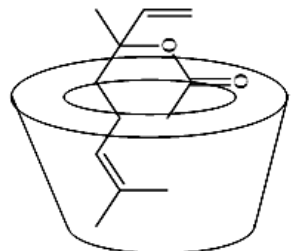

Linalyl acetate in $\beta-C D$

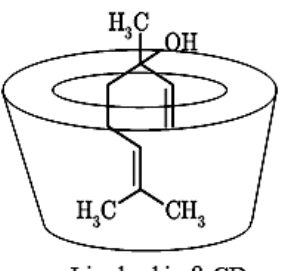

Linalool in $\beta-C D$

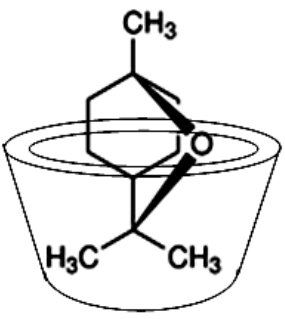

1,8 - Cineole in $\beta-C D$
Fig. 9. Placing of lavender's constituents in the empty cavity of $\beta$-cyclodextrin

$\beta$-Cyclodextrin and its sweat-absorbing property: One of the main aims of choosing $\beta$-cyclodextrin was its sweat absorbing property. Body sweat can be considered as a fatty acid. It is suggested that lavender molecule places in the empty cavity of $\beta$-cyclodextrin and after wearing the fabric which is finished by $\beta$-cyclodextrin containing lavender, fragrance material replaces by short chains of fatty acid (body sweat) and releases (Fig. 10).

\section{Conclusion}

Fragrance materials are volatile compounds and preparing fragrant fabrics with a longer retaining time is a matter of concern. Encapsulation by cyclodextrins is one the best and most secure methods because the treated fabrics will not cause allergy to skin. Four cellulosic fabrics perfumed with different methods by the use of lavender. Due to the presence of four hydroxyl groups on DMDHEU molecule, it can be considered as a chemical binder.

When DMDHEU is placed near salty acids, its oxygen protonizes and DMDHEU loses water, now it can bound with hydroxyl groups of another DMDHEU, cellulose and $\beta$ cyclodextrin. If existed nitrogen in DMDHEU protonizes, formaldehyde will be released. Guest molecule (lavender) can be placed in the cone-shaped hydrophobic cavity of the $\beta$-cyclodextrin and its physico-chemical properties will be changed.

Vapour pressure of lavender decreases and thus its stability against light and air will increase. Best result obtained for second sample because the maximum pick up of lavender took place and also the secondary concentration in second bath was minimum in comparison with other baths.

After 14 days, results of studies indicated that second sample retained more lavender in itself than the others. After 
<smiles>O=C1N(COC2CCCCC2)C(O)C(O)C1(O)C1CC2CCC1C2</smiles>

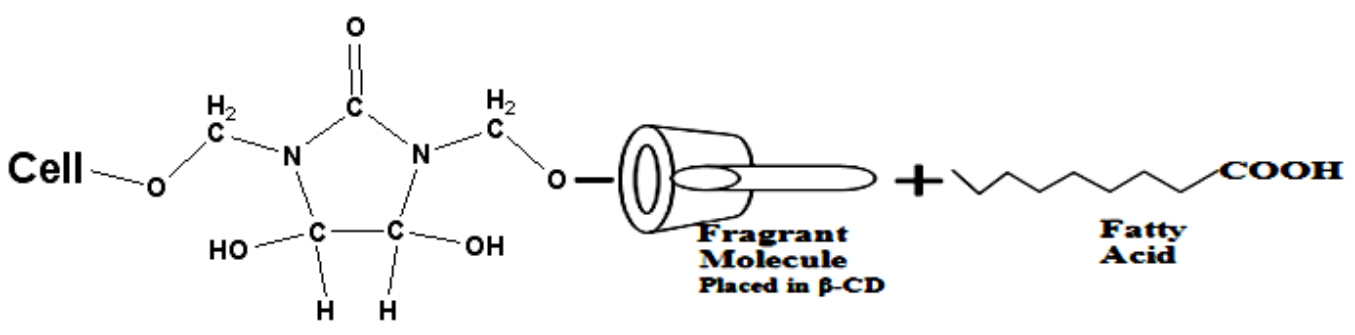

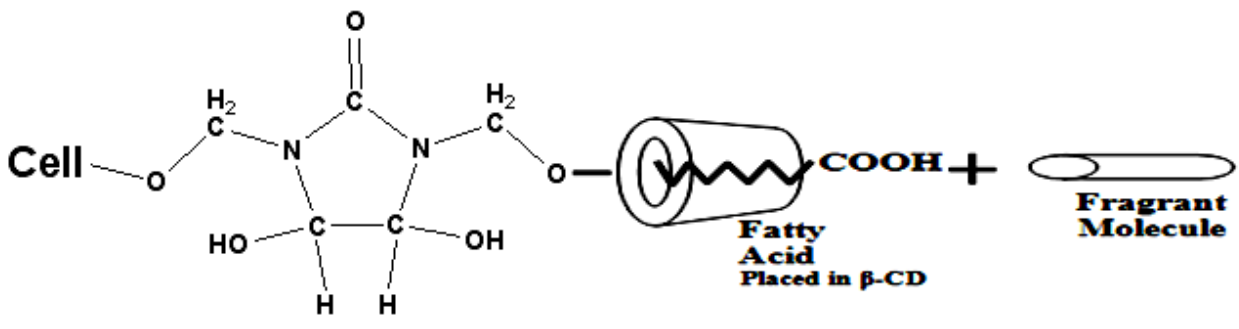

Fig. 10. Replacing fragrance material by fatty acid (body sweat)

studying the amount of lavender, the amount of $\beta$-cyclodextrin which was presented in two baths had been studied by phenolphthalein method. Use of DMDHEU reduces the water sorption in cellulosic fabrics. lavender replaced by body sweat (fatty acid) and released; $\beta$-cyclodextrins' cavity filled by body sweat.

\section{REFERENCES}

1. D. Fiedler, R.G. Bergman and K.N. Raymond, Angew. Chem. Int. Ed., 45, 745 (2006).

2. Á. Piñeiro, X. Banquy, S. Pérez-Casas, É. Tovar, A. García, A. Villa, A. Amigo, A.E. Mark and M. Costas, J. Phys. Chem., B111, 4383 (2007).

3. J. Szejtli, Pure Appl. Chem., 76, 1825 (2004).

4. E.M. Martin Del Valle, Process Biochem., 39, 1033 (2004).

5. H. Cavanagh and J. Wilkinson, Aust. Infec. Coun., 10, March (2005).

6. Hitosh, Hashimoto, J. Incl. Phenom. Macrocycl. Chem., 44, 57 (2002).

7. A. Vyas, S. Saraf and S. Saraf, J. Incl. Phenom. Macrocycl. Chem., 62, 23.

8. M.E. Sangalli, L. Zema, A. Maroni, A. Foppoli, F. Giordano and A. Gazzaniga, Biomaterials, 22, 2647 (2001).

9. T. Loftsson and M. Másson, Int. J. Pharm., 225, 15 (2001).

10. M.H. Lee, K.J. Yoon and S.-W. Ko, J. Appl. Polym. Sci., 78, 1986 (2000).

11. A.G. Grechin, H.-J. Buschmann and E. Schollmeyer, Textile Res. J., 77, 161 (2007).

12. A. Cireli and B. Yurdakul, J. Appl. Polym. Sci., 100, 208 (2006).

13. J. Szejtli, Starch-Stärke, 55, 191 (2003).

14. J. Szetjli, Trends Biotechnol., 7, 170 (1989).

15. B. Voncina and A.M. Le Marechal, J. Appl. Polym. Sci., 96, 1323 (2005).

16. P. Savarino, G. Viscardi, P. Quagliotto, E. Montoneri and E. Barni, Dyes Pigments, 42, 143 (1999).

17. K. Cal and K. Centkowska, Eur. J. Pharm. Biopharm., 68, 467 (2008).

18. T. Loftsson and M.E. Brewster, J. Pharm. Pharmacol., 63, 1119 (2011).

19. T. Loftsson and M.E. Brewster, J. Pharm. Sci., 85, 1017 (1996).

20. M.M.G. El-Sayed Fouda, Ph.D. Thesis, Use of Natural Polysaccharides in Medical Textile Applications, Use of Natural Polysaccharides in Medical Textile Applications, University of Duisburg-Essen, Germany (2005).
21. G. Buchbauer, Perfume Flavorist, 21, 31 (1996).

22. H.-J. Buschmann, Melliand. Textilberchite., 1995(9).

23. H.-J. Buschmann, D. Knittel and E. Schollmeyer, J. Incl. Phenom. Macrocycl. Chem., 40, 169 (2001).

24. M.R. ten Breteler, V.A. Nierstrasz and M.M.C.G. Warmoeskerken, AUTEX Res. J., 2, (2002).

25. P.L. Nostro, L. Fratoni and P. Baglioni, J. Incl. Phenom. Macrocycl. Chem., 44, 423 (2002).

26. N. Gordon, Rev. Prog. Color, 31, 57 (2001).

27. C.X. Wang and Sh. L. Chen, Fibres \& Textiles in Eastern Europe, 13, 41 (2005).

28. C.X. Wang and Sh.L. Chen, Appl. Surf. Sci., 252, 6348 (2006).

29. C.X. Wang and Sh.L. Chen, J. Ind. Textiles, 34, 157 (2005).

30. D. Kut, C. Gunsesoglu and M. Orhan, Fibres \& Textiles in Eastern Europe, 15, 93 (2007).

31. A. Goel and S. Nene, Starch/Starke, 47, 399 (1995).

32. C. Angela, C. Luminita, E. Muresan, M. Corina and R. Butnaru, Romanian Biotechnol. Lett., 15, 5537 (2010).

33. A.M. Pohlit, N.P. Lopes, R.A. Gama, W.P. Tadei and V.F. De Andrade Neto, Planta Med., 77, 598 (2011).

34. M. Poonprasit and S. Pangkamhang, Adv. Mater. Res., 156-157, 311 (2011).

35. B. Boh and E. Knez, Indian J. Fibre Textile Res., 31, 72 (2006).

36. S.K. Vraz, B. Voncina, S.B. Vukušic and D. Katovic, Textile Materials Treated with Eco-friendly Insecticide Agents, ITC and DC: Book of Proceedings of The 4th International Textile, Clothing and Design Conference-Magic World of Textiles, pp. 1067-1072 (2008).

37. C. Luca, A.-M. Grigoriu and A. Grigoriu, Revista de Chimie, 59, 569 (2008).

38. B.T. Ho, D.C. Joyce and B.R. Bhandari, Food Chem., 127, 572 (2011).

39. H.-J. Buschmann, D. Knittel, K. Beermann and E. Schollmeyer, Nachrich. Chem., 49, 620 (2001).

40. R.S. Moon, G.S. Khamkar, P.V. Pede, D.K. Mali and M.V. Kadam, Int. J. Pharm. Technol., 3, 912 (2011).

41. R.V. Pinjari, J.K. Khedkar and S.P. Gejji, J. Incl. Phenom. Macrocycl. Chem., 66, 371 (2010).

42. P.A. Prince and P. Kathiresan, Textile Magazine, 51, 50 (2009).

43. C.N. Choi, T.Y. Hwang, B.K. Ko, R. Kim, S.H. Hong and S.Y. Kim, Polymer, 25, 635 (2001). 